\title{
Clinical and experimental application of the percutaneous twitch superimposition technique for the study of human muscle activation
}

\author{
OM RUTHERFORD, DA JONES, DJ NEWHAM \\ From the Department of Medicine, University College, London, UK
}

SUMMARY The use of percutaneous stimulation for twitch superimposition in the human quadriceps has been compared with stimulation via the femoral nerve. It was found that the relationship between the extra force generated by the twitch and the level of voluntary contraction was independent of the percentage of the muscle stimulated. The technique was used to study activation of the quadriceps and biceps in a group of young healthy adults, and quadriceps activation in patients with musculo-skeletal disorders. The majority of subjects could fully activate their muscles during brief isometric contractions. Inhibition was demonstrated in patients with muscle pain and joint pathology and in subjects with a previous history of knee or joint injury. The technique is easy to use and provides a simple way of detecting inhibition where it might not be anticipated, such as in the absence of pain. Where inhibition is demonstrated the technique can be used to predict approximately the maximum force available.

The assessment of muscle strength in clinical or experimental situations usually requires the full co-operation and motivation of the subject. When people complain of muscle weakness and fatigue the decrement in strength could be due to a loss of muscle tissue, a change in muscle function or an inability or reluctance to activate fully all of the muscle.

In experimental studies of fatigue, electrical stimulation has often been used as a means of distinguishing central and peripheral failure and as a check that the maximal voluntary contractions (MVC) are truly maximal. ${ }^{12}$ The technique has been adapted for clinical use and involves the superimposition of twitches on a maintained voluntary contraction. ${ }^{34}$

With small muscle groups such as the adductor pollicis it is possible to measure the maximum tetanic force by stimulating the motor nerve. However stimulation of the femoral nerve innervating the quadriceps is only practicable with co-operative subjects as the nerve is relatively inaccessible and the generation of maximum tetanic force is rather an alarming pro-

Address for reprint requests: OM Rutherford, Department of Medicine, University College, London, The Rayne Institute, University St, London WC1E 6JJ, UK.

Received 17 January 1986. Accepted 1 March 1986 cedure. For this reason we have compared percutaneous stimulation of part of the quadriceps ${ }^{5}$ with supramaximal stimulation of the femoral nerve as a way of testing for maximum activation. Subsequently percutaneous stimulation has been used to investigate muscle activation in the quadriceps and biceps in normal subjects and for the quadriceps in a group of patients with muscle or bone disorders.

\section{Methods}

Subjects

Quadriceps study A total of 70 subjects were studied for quadriceps activation during maximal voluntary isometric contractions. These comprised 50 young healthy volunteers (40 male, 10 female; age range 17-45 years) of average athletic prowess, eight competing athletes, and 12 patients with a variety of muscle or joint complaints. Details of the patients are given in the table.

Biceps study A total of 18 young healthy volunteers were investigated (10 female, eight male; age range 17-33 years).

\section{Procedures}

Quadriceps study Voluntary isometric contraction force of the quadriceps was recorded with the subject seated in a testing chair. ${ }^{5}$ For percutaneous stimulation two large moistened pad electrodes were placed proximally and distally on the anterolateral thigh. Stimulation was with unidirectional square wave pulses of $50 \mu$ s duration and up to $400 \mathrm{~V}$. The femoral nerve was stimulated at the groin 
Table Measured voluntary isometric force, percentage activation and clinical details of patients studied

\begin{tabular}{|c|c|c|c|c|c|}
\hline Patient & Sex & Clinical details & Voluntary force $(N)$ & $\%$ Activation & Predicted maximum (N) \\
\hline $\begin{array}{l}\text { SR } \\
\text { TV } \\
\text { MB } \\
\text { DG } \\
\text { TT } \\
\text { SS } \\
\text { NP } \\
\text { DJ } \\
\text { MW } \\
\text { RL } \\
\text { MD } \\
\text { HW } \\
\text { GG }\end{array}$ & $\begin{array}{l}\mathbf{F} \\
\mathbf{M} \\
\mathbf{F} \\
\mathbf{M} \\
\mathrm{F} \\
\mathrm{F} \\
\mathbf{M} \\
\mathbf{M} \\
\mathbf{F} \\
\mathbf{M} \\
\mathrm{F} \\
\mathbf{M}\end{array}$ & $\begin{array}{l}\text { Polymyositis } \\
\text { Muscle pain } \\
\qquad, " \\
\text { Dystrophy (LG) } \\
\text { Post-paraparesis } \\
\text { Rheumatoid arthritis } \\
\text {,, , , }\end{array}$ & $\begin{array}{r}174 \\
81 \\
100 \\
60 \\
90 \\
51 \\
376 \\
82 \\
198 \\
101 \\
230 \\
137 \\
169\end{array}$ & $\begin{array}{r}100 \\
100 \\
100 \\
20 \\
20 \\
10 \\
95 \\
30 \\
100 \\
100 \\
100 \\
60 \\
60\end{array}$ & $\begin{array}{l}- \\
- \\
300 \\
450 \\
510 \\
400 \\
273 \\
- \\
- \\
228 \\
280\end{array}$ \\
\hline
\end{tabular}

Maximum isometric force was predicted from the relationship given in fig 3.

where the nerve runs lateral to the femoral artery. A small button electrode on the end of a long handle was used to apply square wave pulses of $50 \mu \mathrm{s}$ duration and up to $200 \mathrm{~V}$. The subjects themselves were responsible for positioning the electrode and maintaining pressure to keep it in place during the experiment.

Biceps study Maximum voluntary isometric force was recorded with the subjects seated in an adjustable straight backed chair with the arm supported on a shelf placed at shoulder height. ${ }^{6}$ The arm and shoulder were secured to prevent lateral or forwards movement. A strap was placed around the wrist and connected by an inextensible cord to a strain gauge attached directly opposite on the wall. Tension was adjusted to keep the elbow at $90^{\circ}$. Percutaneous stimulation was via two small pad electrodes (approx $30 \mathrm{~cm}^{2}$ ) with the same duration pulse of up to $200 \mathrm{~V}$.

\section{Protocols}

The study comprised three parts: (1) in the quadriceps a comparison of percutaneous twitch superimposition with stimulation via the femoral nerve during submaximal and maximal contractions. This was combined with the determination of the relationship between the extra force generated by the twitch and the level of voluntary force. (2) A study of activation of the quadriceps during maximal voluntary contractions (MVC) in healthy subjects and patients with musculo-skeletal disorders. (3) A study of biceps activation during maximal voluntary contractions in young healthy subjects.

(1) For percutaneous stimulation the voltage was adjusted to maximally stimulate different proportions of the muscle comparing the tetanic force at $30 \mathrm{~Hz}$ (T30) with the MVC. When stimulating via the femoral nerve the voltage was increased until the twitch height plateaued and was then increased by a further $10 \mathrm{~V}$ to ensure supramaximal stimulation. Subjects were requested to perform either maximal contraction or randomly selected submaximal contractions during which twitches were superimposed at $1 \mathrm{~Hz}$. When a series of contractions was studied sufficient time was left between each contraction to allow the potentiated twitch to recover to the control height recorded at the beginning of the experiment.

(2 and 3) The protocol used to study activation during maximal contractions was the same for both the quadriceps and biceps. Subjects first performed three MVCs and the highest was recorded. Stimulation voltage was adjusted during a $1 \mathrm{~Hz}$ train to between 350 and $400 \mathrm{~V}$. The subjects were then asked to perform another MVC and maintain it for approximately 4 seconds during which twitches were superimposed.

\section{Results}

In normal subjects there is a progressive decline in the extra force generated by the twitch as the voluntary force increases to maximum (fig la). During a truly maximum contraction no extra force is generated by the twitch, and in many cases there can be a momentary drop in force immediately after the stimulus.

To investigate whether twitch potentiation was occurring during the contraction, twitch height was measured during a series of submaximal contractions of the quadriceps in one subject. Three levels of submaximal contraction were studied: 20,40 and $60 \%$ of maximum. On each occasion twitch height was measured prior to the contraction. The subject then contracted up to the required level, as indicated by a visual target, and this was held for $5 \mathrm{~s}$ with twitches superimposed. Twitch height was recorded immediately after the contraction and then at regular intervals until recovery to the pre-contraction level. Results are given in fig 2 . Twitch height is expressed as

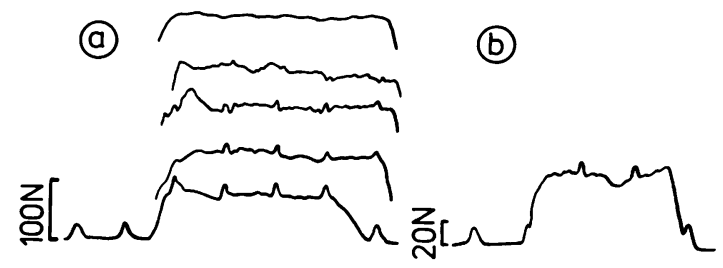

Fig 1 Extra force generated by superimposed twitches: (a) during progressively larger voluntary contractions in a normal healthy subject and $(b)$ in a patient with muscle pain showing a large amount of inhibition. 


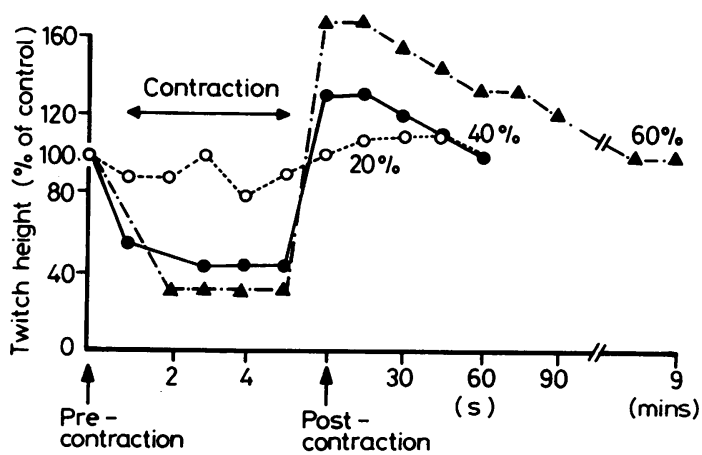

Fig 2 Twitch height (as a percentage of control) during and after a $5 s$ voluntary contraction.

a percentage of the pre-contraction height. There was no evidence of potentiation of the twitch during the contraction. Immediately after each contraction the twitch was potentiated by different amounts depending on the contraction level. Recovery of twitch height was complete after 1 min for the 20 and $40 \%$ contractions and after $8 \mathrm{~min}$ for the $60 \%$.

The relationship between the extra force generated by the twitch and the level of voluntary force Four subjects were studied of which two had femoral nerve stimulation. Voltages were adjusted to stimulate $20-60 \%$ of the quadriceps percutaneously and the whole muscle via supramaximal femoral nerve stimulation.

For each subject, on each occasion tested, a curve was fitted by eye to the results and a composite curve constructed from these (fig 3 ). In our original report of this work ${ }^{7}$ we represented this relationship as being linear; however, with the expanded results it would appear to be more curvilinear. There was no significant difference in the relationship given by femoral nerve stimulation (open circles, fig 3 ) compared with that for percutaneous stimulation (closed circles, fig 3). There was also no significant difference between percutaneous stimulation of different percentages of the muscle, as measured from the $T_{30}$, in the range studied $(20-60 \%)$.

Activation of quadriceps in normal subjects and patients Fifty normal subjects were tested for quadriceps activation during an attempted MVC during which five showed incomplete activation. Of these five, two had suffered fractures of the femur in the previous year and the subject with the largest decrement (activating $75 \%$ of the muscle) had bony spurs on each ankle which caused much pain and had severely reduced his exercise level. Of the eight athletes studied all could maximally activate their muscle.
Results and clinical details for the patients studied are given in the table. Reduced activation was seen in those patients with muscle pain and joint pathology. Patients with extensive loss of muscle tissue as a result of disease or paraparesis were still able to activate fully their muscle. A tracing of a record from a subject with muscle pain is given in fig $1 \mathrm{~b}$.

Activation of biceps Eighteen normal healthy subjects were studied for biceps activation during an isometric contraction. Five were found to be unable to activate fully with most showing very small decrements such that the extra force was barely visible on top of the voluntary force.

\section{Discussion}

We have examined the ability of patients and healthy subjects to generate the maximum force from all available motor units during voluntary isometric contractions. This has been investigated using an adapted twitch superimposition technique such that motor units not recruited during the voluntary effort will give an observable twitch response on stimulation.

The maximum voluntary force of the quadriceps is a measurement commonly made both in clinical and experimental studies. It is therefore important to be able to check routinely the activation of the quadriceps during a maximal contraction. Although stimulation of the femoral nerve is possible it is not a technique that can be used routinely with inex-

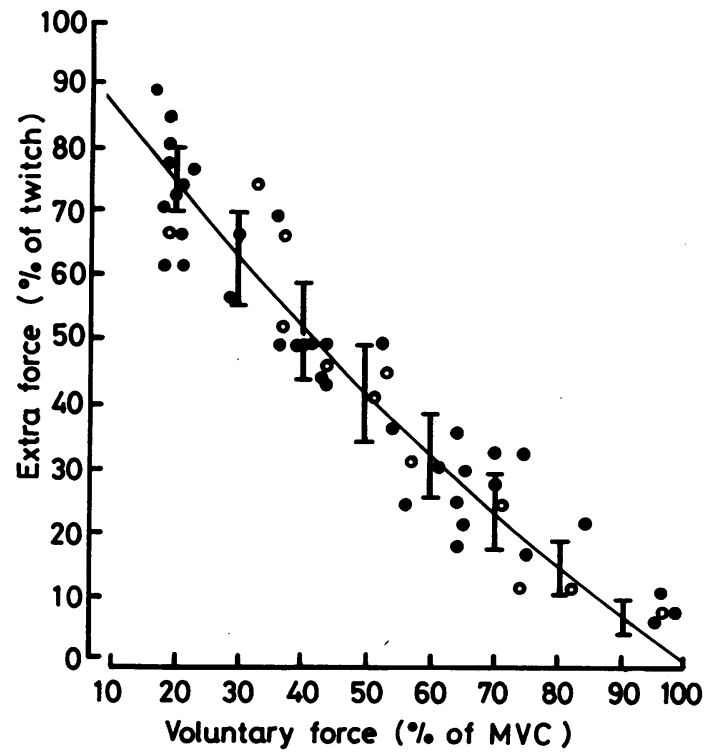

Fig 3 Relationship between extra force generated by the superimposed twitch and level of voluntary contraction force. $(\bigcirc$, femoral nerve stimulation; $\bigcirc$, percutaneous stimulation.) 
perienced subjects. However percutaneous stimulation is a simple and acceptable technique which can be used to activate up to $60 \%$ of the muscle as measured from the $T_{30}$. The relationship between the decrement in twitch force and voluntary force was the same when using percutaneous or femoral nerve stimulation, indicating that percutaneous stimulation is a valid test of activation (fig 3 ).

It was interesting to note that there was no potentiation of the twitch during the contraction. However the first twitch after the contraction was potentiated. The time taken for the twitch height to return to precontraction level varied depending on the level of voluntary contraction (fig 2).

In the clinical situation the technique can be used to distinguish between central and peripheral causes of weakness. This has previously been used to study functional weakness of the calf muscles in two patients. ${ }^{8}$ McComas et $\mathrm{al}^{8}$ demonstrated a large decrement in activation similar to that seen in this study for patients with muscle pain and joint pathology. We were able to distinguish three patient groups: those with pain and incomplete activation, those with inhibition without pain and those with weakness as a result of loss of muscle tissue who were able to activate all the muscle available. In everyday activities the last of these groups are probably working at near maximal forces just to walk and climb stairs and as a result are practised at using all the muscle available. The decreased activation shown by some patients may be caused by several factors. The patient may consciously not try as hard as possible thereby reducing pain, alternatively reflex inhibition from the affected joint or muscle may prevent maximum force production without any conscious perception of pain. The percutaneous stimulation technique is particularly useful for identifying inhibition in the absence of pain.

Of the five apparently healthy subjects who showed inhibition of the quadriceps it subsequently transpired that three had limited exercise capacity in the previous 12 months as a result of injury or joint problems. None experienced any pain during the contraction. As all appeared highly motivated it is likely that some form of reflex inhibition was occurring. Inhibition of the quadriceps from non-painful but damaged knees is a well recognised phenomena. ${ }^{9-11}$

In the past it has been a widely held belief that one of the major adaptations during strength training is increased activation of the muscle resulting in a greater force per unit area. ${ }^{1213}$ This assumes that prior to training normal subjects are unable to activate maximally their muscles. The present results and those of other studies ${ }^{314}$ contradict this, at least for brief contractions. There would, however, appear to be a difference in the ability of subjects to activate certain muscles. In this study a greater percentage of the subjects were unable to activate fully their biceps (nearly $30 \%$ of subjects tested). Belanger and McComas $^{3}$ found that $50 \%$ of subjects were unable to activate fully the plantar flexors of the foot, as opposed to only one subject for the dorsiflexors. This difference between muscles may be due to a difference in their general pattern of use. The quadriceps may be involved in more maximal movements than the biceps during everyday activities. In view of this it is of interest that strength training of the biceps is reported to result in greater strength gains per day than for the quadriceps, possibly reflecting its relatively detrained state. $^{15}$

\section{References}

${ }^{1}$ Merton PA. Voluntary strength and fatigue. $J$ Physiol (Lond) 1954;123:553-64.

${ }^{2}$ Bigland-Ritchie B, Jones DA, Woods JJ. Excitation frequency and muscle fatigue: electrical responses during human voluniary and stimulated contractions. Exp Neurol 1979;64:414-27.

${ }^{3}$ Belanger AY, McComas AJ. Extent of motor unit activation during effort. J Appl Physiol 1981;51(5):1131-5.

${ }^{4}$ Bellemere F, Woods JJ, Johansson R, Bigland-Ritchie B. Motor-unit discharge rates in maximal voluntary contractions of three human muscles. $J$ Neurophysiol 1983;50(6):1380-92.

${ }^{5}$ Edwards RHT, Young A, Hosking GP, Jones DA. Human skeletal muscle function: description of tests and normal values. Clin Sci 1977;52:283-90.

${ }^{6}$ Jones DA, Newham DJ. A simple apparatus for eccentric or concentric exercise of the biceps. J Physiol (Lond) 1985;365:10P.

${ }^{7}$ Chapman SJ, Edwards RHT, Greig C, Rutherford O. Practical application of the twitch interpolation technique for the study of voluntary contractions of the quadriceps in man. J Physiol (Lond) 1984;353:3P.

${ }^{8}$ McComas AJ, Kereshi S, Quinlan J. A method for detecting functional weakness. J Neurol Neursosurg Psychiatry 1983;46:280-2.

${ }^{9}$ Blockey NJ. An observation concerning the flexor muscles during recovery of function after dislocation of the elbow. J Bone Joint Surg 1954;36A:833-40.

${ }^{10}$ de Andrade JR, Grant C, Dixon A St J. Joint distension and reflex muscle inhibition in the knee. $J$ Bone Joint Surg 1965;47A:313-22.

${ }^{11}$ Stokes M, Young A. The contribution of reflex inhibition to arthrogenous muscle weakness. Cli Sci 1984;67:7-14.

${ }^{12}$ Moritani T, deVries HA. Neural factors versus hypertrophy in the time course of muscle strength gain. Am J Phys Med 1979;58:115-30.

${ }^{13}$ Ikai M, Fukunaga T. A study on training effect on strength per unit cross-sectional area of muscle by means of ultrasonic measurement. Int $Z$ angew Physiol 1970;28:173-80.

${ }^{14}$ Edwards RHT, Hill DJ, Jones DA. Heat production and chemical changes during isometric contractions of the human quadriceps muscle. $J$ Physiol (Lond) 1975;251:303-15.

${ }^{15}$ Darcus HD, Salter N. The effect of repeated muscular exertion on muscle strength. $J$ Physiol (Lond) 1955;129:325-36. 